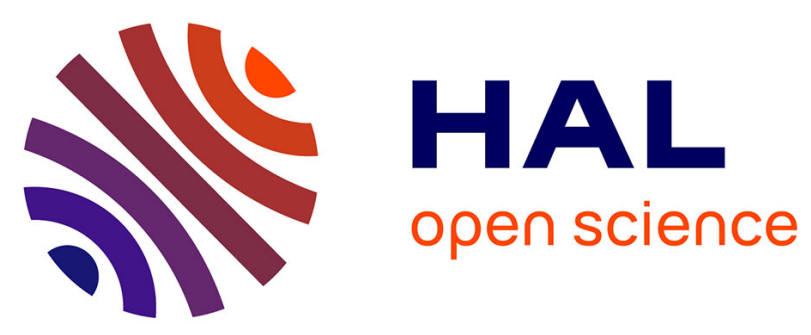

\title{
Forcer le destin à la verticale
}

Gilles Raveneau, Alain Robert

\section{- To cite this version:}

Gilles Raveneau, Alain Robert. Forcer le destin à la verticale. Ethnologie française, 2006, Les sports à risques, 36 (4), pp.690-692. 10.3917/ethn.064.0690 . hal-03134837

\section{HAL Id: hal-03134837 \\ https://hal.parisnanterre.fr/hal-03134837}

Submitted on 8 Feb 2021

HAL is a multi-disciplinary open access archive for the deposit and dissemination of scientific research documents, whether they are published or not. The documents may come from teaching and research institutions in France or abroad, or from public or private research centers.
L'archive ouverte pluridisciplinaire HAL, est destinée au dépôt et à la diffusion de documents scientifiques de niveau recherche, publiés ou non, émanant des établissements d'enseignement et de recherche français ou étrangers, des laboratoires publics ou privés. 


\section{Forcer le destin à la verticale}

Propos recueillis par Gilles Raveneau

Alain Robert

grimpeur

Alain Robert est né le 7 août 1962 à Digoin, en Saône-et-Loire. Il est marié et père de trois enfants. Il passe son enfance à Valence. C'est à cette époque qu'en regardant le film La neige en deuil il découvre que des hommes cherchent à gravir des montagnes en se jouant de la verticalité et du vide. C'est une révélation et la confirmation des valeurs de courage et de bravoure qui ont bercé ses jeux de petit garçon. Initié à l'escalade par les scouts, il va bientôt s'entrainer sur les falaises des environs de Valence avec un copain guère plus aguerri que lui. Ensemble, ils affinent sur le tas les rudiments appris dans les camps scouts. Il devient vite un bon grimpeur. Il gravit quelques-unes des faces les plus difficiles des gorges du Verdon et des Alpes (face nord des Droites, traversée des arêtes de l'Oisans). Mais en 1982, à dix-neuf ans, deux accidents le stoppent dans son élan. Le second, en septembre, est très grave. Il fait une chute de quinze mètres sur une dalle en calcaire et se retrouve cinq jours dans le coma avec des fractures multiples (crâne, nez, poignets, coude, bassin et talons) et un problème d'oreille interne (vertige). Le pronostic des médecins est formel : il ne pourra plus jamais grimper. La Sécurité sociale le considère comme invalide à $60 \%$ et lui propose une indemnité COTOREP (Commission technique d'orientation et de reclassement professionnel) pour son handicap. Il refuse et va s'ingénier à montrer aux médecins qu'ils ont tort. Deux ans après, il escalade à nouveau les falaises et dépasse les performances qui étaient les siennes avant l'accident.

En 1988, il s'engage plus radicalement encore dans l'escalade en solo, sans corde ni artifice, qu'il pratique déjà depuis 1977. Il grimpe d'abord encordé pour repérer les gestes et les prises, puis, après quelques essais, se lance sans matériel. Il s'impose progressivement comme le plus grand spécialiste du solo intégral en falaise. Il gravit alors une quinzaine de voies de niveau 8 (le plus haut degré de difficulté). Il réalise par exemple en octobre 1996 l'escalade à mains nues de "Pol Pot» dans les gorges du Verdon, une voie extrême qu'ouvrit et gravit encordé Patrick Edlinger ; ou encore celle du "Rêve de papillon " et de "La nuit du cauchemar » sur les falaises du Buoux, l'enchaînement de "L'abominafreux " et de "L'abominable homme des doigts " près de Cornas. Patrick Edlinger lui remet le prix de la Performance sportive au Festival de Janssens en 1991. En 1993, il reçoit le prix du Comité international olympique pour la performance d'un athlète. La même année, il établit un nouveau record du monde de la difficulté en escalade solo.

Cette reconnaissance sportive lui permet d'être remarqué par la société italienne d'horlogerie Sector, qui concentre son action de sponsoring sur les sports de l'extrême'. Elle lui propose de faire un documentaire dans lequel il doit grimper un gratte-ciel à mains nues. L'année 1994 marque un nouveau tournant dans sa vie. Il escalade sa première tour à Chicago. Ce sont ses débuts de grimpeur urbain qui vont le faire connaître du grand public. Ses ascensions illégales le conduisent plusieurs fois en prison. Il enchaîne les immeubles aux quatre coins de la planète et devient pour les médias Spiderman, l'homme-araignée ${ }^{2}$. À partir de cette date, il devient professionnel et se spécialise dans l'escalade urbaine. En une dizaine d'années, Alain Robert a gravi plus de quatre-vingts gratte-ciel et grands monuments à travers la planète dont les plus hautes tours du monde à plus de 400 mètres de haut (les Petronas twin towers à Kuala Lumpur en 1997, la Sears tower à Chicago et Tapei 101 à Taipei en 2004).

Plus on fait du sécuritaire dans nos sociétés et plus on réduit l'espace de liberté des individus. Ma position est celle d'un homme libre. Et cette liberté peut m'envoyer en prison parce que je décide de grimper un immeuble à mes risques et périls. C'est quand même incroyable ! Pour avancer dans la vie, il faut prendre des risques au sens large du terme. Évidemment, cela ne cadre pas du tout avec le discours politique d'aujourd'hui. Mon message va à l'encontre de la société actuelle, hypersécuritaire. Je suis né avec des valeurs de bravoure et de courage, comme les chevaliers du Moyen Âge, Robin des bois et Zorro. C'est vrai qu'au sujet de ma récente tentative d'ascension illégale à Houston, au Texas ${ }^{3}$, ce n'est pas Alain Robert qui voulait aller grimper ce building, mais Zorro ou Robin des bois ! C'est une histoire d'amour-propre et de revanche contre la bêtise. Ce building n'a rien de particulier, j'en ai grimpé des plus difficiles aux États-Unis.

J'aime les choses exigeantes. La recherche est toujours la même, en falaise comme sur les gratte-ciel. Ce que j'aime dans l'escalade, c'est l'engagement. Le risque est un moteur pour moi. C'est une clé. Lorsque j'étais gamin, j'étais très peureux, mais je rêvais d'être un chevalier. C'est ce qui prouve qu'on peut être maitre de son destin : peureux, introverti, mal dans sa peau et se construire ce dont on a rêvé. Prendre des risques, c'est être brave, "avoir des couilles " comme on dit. Maintenant, le courage pour moi, ce peut être aussi l'action de sœur Emmanuelle ou de l'abbé Pierre. Des formes de courage qui sont plus utiles à l'être humain que ce que je fais.

Quand j'escalade, je peux tomber. C'est «ma peau » que je mise. Prendre des risques est une décision que tu 


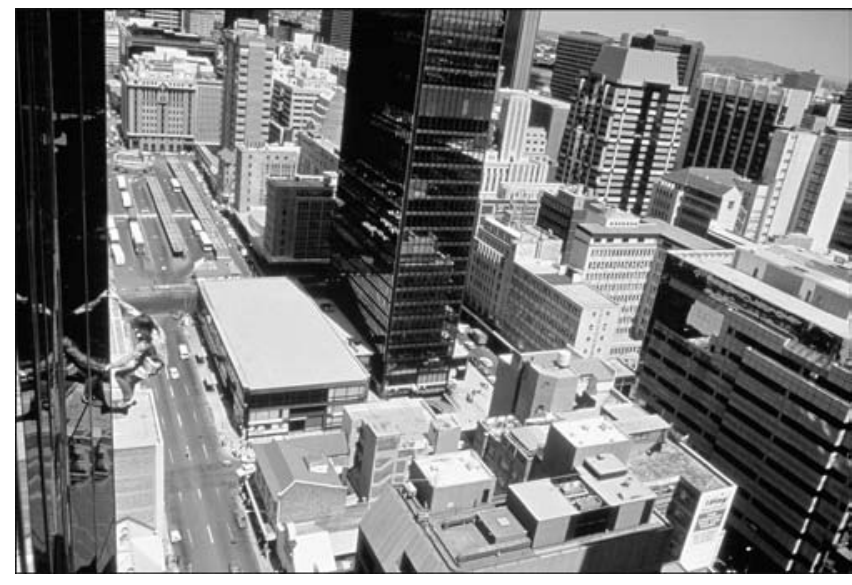

1. Tour IBM à Johannesburg en 1998 (C) Alain Robert).

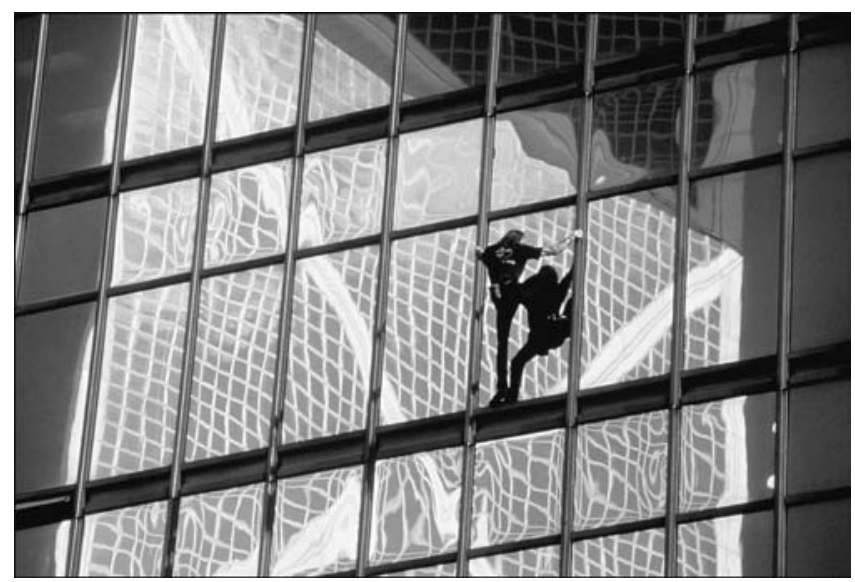

2. Far East Finance Center à Hong-Kong en 1996 (C) Alain Robert).

prends en connaissance de cause, avec un savoir, une expérience et une marge de manœuvre. Quand je grimpe un immeuble trois fois, le risque redescend au niveau zéro. Avec le temps, tu apprends à négocier avec le vide. Ce qui peut poser problème, c'est la difficulté, le fait de flirter avec tes limites. Je l'ai fait en falaise et sur gratte-ciel. Là, tu peux très bien tomber. Cela ne tient à pas grand-chose le fait de réussir ou d'échouer. Ce qui est intéressant, c'est le jour J où tu grimpes sans corde. Tu dois en faire abstraction. Mais si tu montes avec beaucoup de marge, que tu grimpes réfléchi, à un rythme correct, tu ne prends pas de risque.

Je n'ai pas peur. Dès que je commence à grimper, je suis concentré sur ce que je fais. La peur, je l'éprouve avant une ascension, pas pendant. Lorsque tu escalades, tu as autre chose à faire pour arriver en vie. Tu évacues la peur, elle n'est plus présente à l'esprit. Je ne suis pas un yogi et je ne fais pas de la méditation. Au contraire, avant une ascension, les gens veulent des autographes,

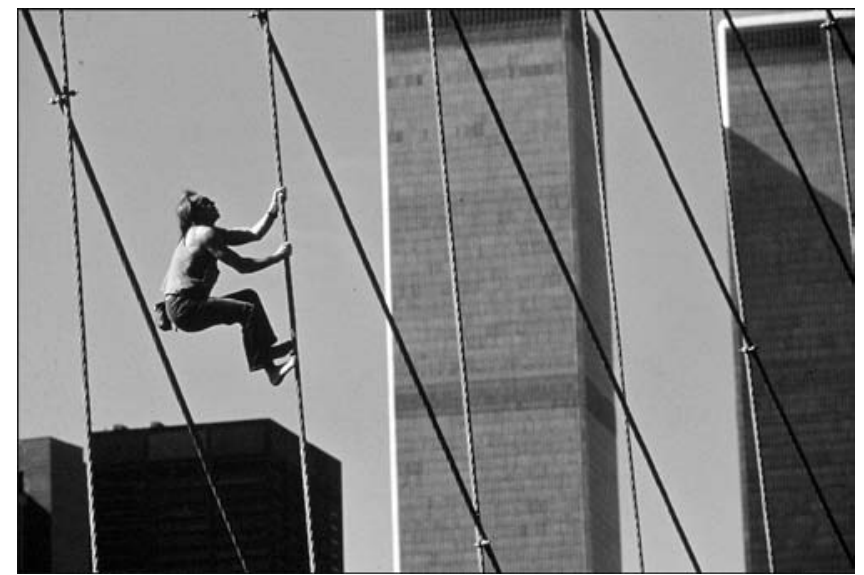

3. Le pont de Brooklyn en 1994 à New York avec, en fond, les Twin Towers détruites en 2001 (C) Alain Robert).

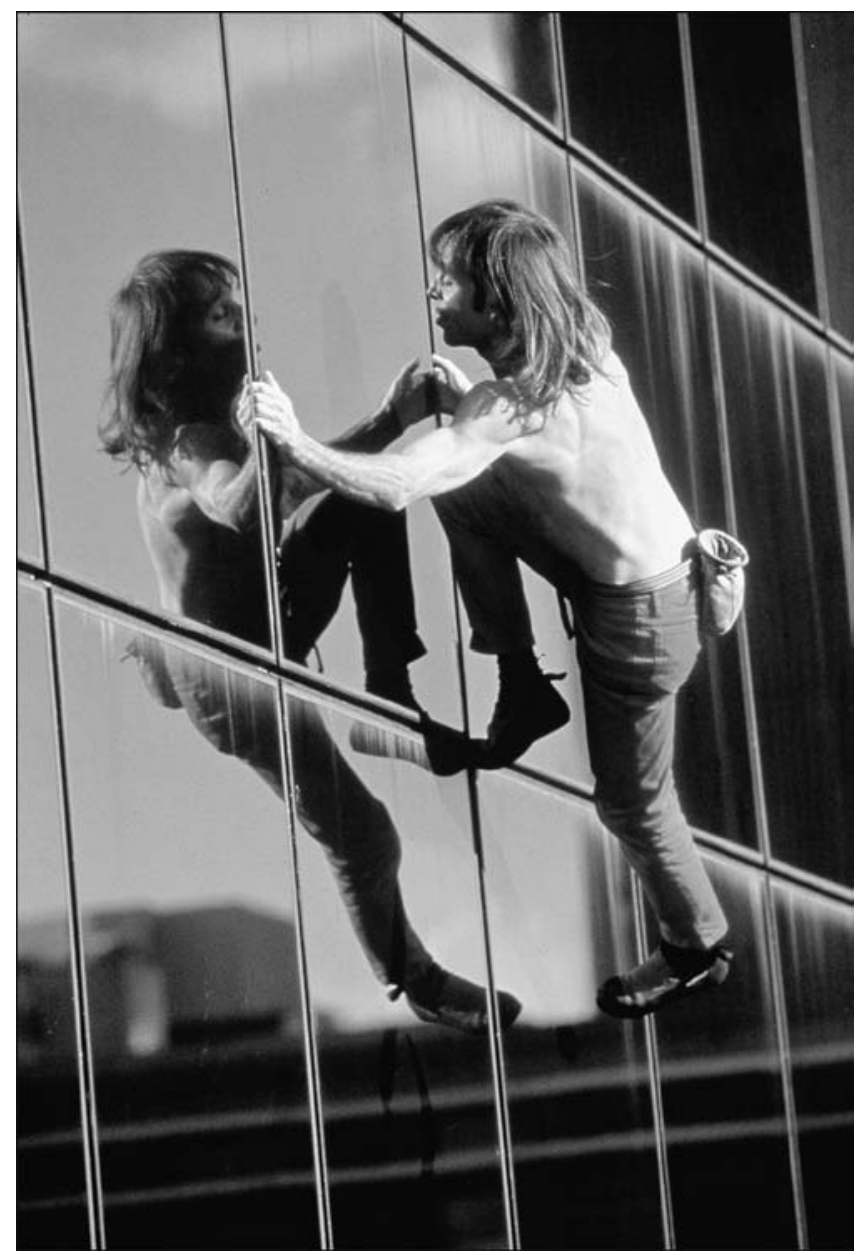

4. Le Paramount Building à New York sur Times Square en 1994 (C) Alain Robert). 
des photos. Ils n'analysent pas du tout que j'aurais besoin d'être au calme.

Les immeubles, ce n'est pas mon idée au départ, mais celle de la société Sector. Quand ils ont évoqué les gratte-ciel, je me suis demandé de quoi ils parlaient! Je n'habitais pas en ville, je n'avais jamais regardé une tour. Ils m'ont payé des voyages pour que j'étudie les structures. J'ai transposé aux gratte-ciel ce que je savais faire sur les falaises; mais le pas était énorme. Au départ, j'étais un peu effrayé. Je trouvais les tours impressionnantes, très lisses et je ne savais pas comment $j$ 'allais me comporter sur ce type de structure artificielle. Dès la première aventure, j'ai trouvé ça très excitant, fascinant même. Il n'y avait plus seulement l'escalade, mais l'illégalité, l'arrestation, la prison, les médias... L'image du grimpeur urbain a fini par prendre le dessus. Je me suis retrouvé dans un univers plus complet qui m'a ouvert sur le monde entier, les gens, les voyages, les rencontres. Le public en redemande. Je reçois énormément d'e-mails. J'ai un site électronique qui tourne à mille visiteurs par jour et quinze mille quand il y a de l'actualité. Les gens sont admiratifs, les messages sympas. Tu as la sensation de faire une chose que les autres ne font pas. Et la preuve, ce sont tous ces gens qui m'écrivent pour dire que je leur apporte quelque chose : le courage d'aller plus loin, de repousser leurs limites, le fait de leur servir de modèle ou de les faire rêver. Tu as alors le sentiment d'être utile.

Je n'ai jamais pensé que cela se passerait comme ça. J'ai tout de suite aimé. Cela a radicalement changé ma vie. Au lieu de travailler dans un magasin de sport à mi-temps pour pouvoir continuer à pratiquer l'escalade, je suis passé professionnel à temps plein. C'est devenu une façon de gagner ma vie et d'obtenir une reconnaissance que j'avais seulement dans les milieux spécialisés.

J'ai eu sept accidents. J'ai été condamné assez tôt dans ma vie par les médecins et finalement j'ai toujours eu ce désir de continuer à faire de l'escalade, même avec un taux d'incapacité élevé. C'est triste d'être à la COTOREP. À dix-neuf ans, tu aspires à réaliser tes rêves. Lors de tous mes accidents, je me suis reconstruit très vite. On est maître de son destin. Libre de choisir une route et si elle apparaît mauvaise, de décider d'en prendre une autre, et ainsi de suite. Tant qu'on n'est pas mort, on peut se tromper, changer et prendre le bon chemin un jour. Mais il faut avoir l'envie d'essayer et ne pas penser que tout est déjà écrit. Curieusement, je suis devenu plus fort après mes accidents. Le fait que les médecins aient estimé que ma carrière était derrière moi m'a donné envie de leur montrer qu'ils avaient tort et qu'elle était bien devant. C'est une volonté de vivre. C'est toi qui décides de ce que tu veux faire de ta vie.

Je suis reconnu pour faire des choses qui sont exceptionnelles. Mais il y a d'autres personnes qui sont reconnues simplement parce qu'elles passent à la télévision. Quelle gloire y a-t-il à passer à la télé ?! Parce que tu apparais sur le petit écran, tu deviens tout à coup quelqu'un qui existe? Mais avant que je sois médiatisé, je grimpais déjà à un très haut niveau. Il y a vingt ans, je m'engageais de la même façon en solo sur des falaises. Mais je n'étais pas connu du grand public. Les raisons, hier comme aujourd'hui, sont les mêmes : le plaisir de grimper.

\section{Notes}

1. Elle a ainsi financé : Gérard d'Aboville en 1991, pour sa traversée du Pacifique à l'aviron en solitaire et sans assistance ; Borge Ousland en 1994, qui devient le premier homme à atteindre le pôle Nord à pied et sans assistance ; Umberto Pelizzari, premier homme à atteindre la profondeur de 150 mètres en plongée en apnée; ou encore Mike Horn en 2000 , qui réalise un tour du monde sur la ligne de l'équateur sans jamais utiliser de véhicule à moteur.

2. Personnage créé en 1962 par Stan Lee et Steve Ditko, Spiderman est l'un des personnages les plus populaires de l'univers des comics.

3. Au moment de cet entretien, il est sommé de retourner au Texas pour faire trois jours de prison suite au jugement d'une de ses tentatives avortées d'ascension à Houston. Il avait été arrêté avant même d'avoir pu grimper. 